\title{
Mullerian Duct Defect: Uterus Didelphys
}

\author{
Article by Daniel Josh Kanhai, Ashley Dudhnath \\ Medical students, College of Medicine, Texila American University, Guyana, South America
}

\begin{abstract}
This report is centered around a 20 years old patient with uterus didelphys who has successfully conceived, carried her pregnancy to term and delivered her twins by lower segment caesarean section as requested, without any significant complications. The best diagnostic modality will also be discussed and its importance in the next management step or surgical intervention.
\end{abstract}

Keywords: Mullerian duct defect; Congenital anomaly; Uterus Didelphys.

\section{Introduction}

Mullerian anomalies are one of the most alluring disorders seen in the obstetrician and gynecology department, especially ones that are on the rare end of the spectrum. The Mullerian ducts are the beginning of the female reproductive system which proliferates because the sex gene does not accommodate the production of testosterone. The ducts undergo fusion to form the uterine cavity with the two (2) fallopian tubes (one on each side), the cervix and the upper on third (1/3) of the vagina.

Uterine anomalies develop as the normal fusion of the ducts are hampered and agenesis, hypoplasia or both occurs. These uterine anomalies are classified into seven classifications (0-VI) for easy differentiation. Mullerian duct anomalies are often associated with renal and axial skeletal anomalies, and are usually discovered when patients are examined for another condition. Mullerian duct anomalies are usually asymptomatic and the normal sexual development of the female masks the internal problem with the uterus. This normal development and a wide variety of clinical presentation makes the diagnosis difficult, however once it is diagnosed there are several treatment options to provide the patient with that will be tailored to the specific defect. ${ }^{[1]}$

The incidence of the Mullerian duct defect depends on the authors and most reported an incidence of $0.1-3.5 \%{ }^{[2]}$ In 2001, Grimbizis GF, Camus $M$ et al obtained an incidence of $4.3 \%$ in the female population (complied 5 studies $=3000$ women); $3-6 \%$ of women with infertility have an anomaly; 5 $10 \%$ of women with recurrent abortions have an anomaly, mostly in $3^{\text {rd }}$ trimester. Also, a Danish study of 622 women (20-74 years old), all of whom were examined with saline contrast sonohysterography, suggested the prevalence of Mullerian duct anomalies in the population to be 9.8\%; the prevalence was particularly high in nulliparous women and in those with oligomenorrhea. Grimbizis GF, Camus $\mathrm{M}$ et al also had a prevalence of $4.3 \%$ in general population, $3.5 \%$ in infertile women and $13 \%$ with recurrent pregnancy loss. ${ }^{[2]}$

Uterus Didelphys is a class III Mullerian defect that comes about when the midline fusions of the ducts cease forming either complete or incomplete. This event causes the formation of two independent uterine cavities each with single horns and two cervixes. In $75 \%$ of cases, there can be a septate vagina giving the impression of two vaginas. ${ }^{[4]}$ Women with Didelphys uterus are usually asymptomatic unless an obstruction is present, with symptoms being hematometrocolpos, hematometra and hematosalpinx.

As mentioned above, there must be suspicion of renal anomalies; as there was a $20 \%$ chance recorded. ${ }^{[2]}$ Some other parallel anomalies include bladder exstrophy with or without vaginal hypoplasia, congenital vesicovaginal fistula with hypoplastic kidney and cervical agenesis.

Patients with this condition are not infertile and therefore are able to become pregnant. Twin pregnancy is quite rare. It is usually di-zygotic, di-chorionic and di-amniotic in independent uterine cavity. There are amazing cases where the second fetus is delivered after several days or weeks suggesting that the pregnancy can occur at separate times. However, lactation may only occur until the second fetus is delivered. 
The obstetric outcome data of a patient with uterus didelphys is very minimal but can include unilateral placental abruption or unilateral preterm labor. ${ }^{[2]}$ The management of delivery for these patients can be tricky and a choice between lower segment caesarean section (LSCS) or spontaneous vaginal delivery must be made.

\section{Case report}

A 20years old G1P0 was first admitted on 20/02/2017 with a chief complaint of lower abdominal pain and lower back pain with frequent contractions and passing a big clot and slime. She is presenting with Dichorionic Diamniotic twin gestation at 32 weeks and $32^{+2}$ weeks $\left(15^{+1} \mathrm{USG}\right)$, preterm contractions, vaginal candidiasis and not in labor.

The patient was treated and had no complaints of contractions or any pain and so was discharged on 22/07/2017. On fetal non-stress test twin 1 was cephalic and twin 2 was breeched. She then presented to the hospital again on 10/08/2017 complaining of feeling a gush of greenish fluid in the washroom. On examination there were abundant vaginal candidacies and greenish cream secretions on speculum. The impression at admission was 20years old G1P0 @ $35^{+5}\left(14^{+6}\right.$ USG) Dichorionic Diamniotic twins with vaginal sepsis and preterm premature rupture of membranes was ruled out. The patient was treated and discharged the following day.

On 23/08/2017 the patient was at $37^{+6}$ gestation complaining of contractions every 20 minutes, greenish discharge and decreased fetal movement. On 24/09/2017 the patient was admitted for lower segment caesarean section (LSCS). She says that there is fetal movement and no show. On 25/09/2017 the LSCS was done and two intrauterine contraceptive devices were placed. Post LSCS the patient complained of lower abdominal pain and shortness of breath. On the 26/08/2017 the patient developed acute urinary retention secondary to inadequate pain management. The patient was treated and observed for the next few days. On the 29/08/2017 the Patient was discharged and told to follow up in clinic.

\section{Discussion}

Uterine Didelphys can present as an asymptomatic uterine anomaly as was shown in this case report. The patient had no abnormal menstrual pattern since menarche and there were no clinical manifestations of any abnormalities present within the patient. No manifestations were present because this patient had no obstruction (septum) in the vagina to cause any bleeding or interruption on coitus or cause any of the three: hematometrocolpos, hematometra or hematosalpinx to occur and the patient was not infertile.

Upon LSCS, a live baby boy was extracted at $38+1$ weeks at $3100 \mathrm{~g}$ and then a live baby girl was extracted at 38+1 weeks at 2800g. Also, both neonates had an APGAR score 9, 10, 10. The neonates had no complications and were both delivered at term. They had no restriction nor were they small for their gestational age. This proves that there is no restriction to the growth of the fetus by the hemiuteri and as believed that the smaller cavity can contribute to preterm deliveries and small neonates were not applicable to this case.

To add to the LSCS events, the shape of the uterus was heart shaped which suggested a bi-cornate uterus, but on exploration the septum was noted and the double cervixes confirming uterus Didelphys. The misconception in the beginning of the case is possible because both mullerian defects have the same shape of uterus. The trans- abdominal ultrasonographies performed did not detect the uterine anomaly during pregnancy because of the thin septum between the two cavities. The MRI is mostly used to give a concrete diagnosis of a uterine anomaly and while at it, will also show any other associated renal anomalies that may be present. ${ }^{[6]}$ Transvaginal sonography is also another great diagnostic tool for uterine anomalies. ${ }^{[6]}$ The sensitivity and specificity of MRI is $100 \%$ while the endovaginal sonography(EVS) has a sensitivity of $100 \%$ and a specificity of $80 \% .{ }^{[6]}$ Both MRI and EVS have a $100 \%$ sensitivity and specificity in distinguishing those uterine anomalies that need repairs. These high values actually decrease the rate at which an invasive method of diagnosis is required. 
When the diagnosis has been made and it is either complete or incomplete, a specific management will be considered for the patient. In this patient's case the uterus was giving the patient no problems so there was no need for any form of management.

However, it is advised that she should definitely be brought back to the institution to undergo an MRI scan or even a trans-abdominal ultrasonography so that any congenital anomaly can be ruled out since they are common in patients especially with uterus didelphys. Her daughter should also be screened for any mullerian duct anomalies as well as renal anomalies as it was noted to be a genetic relation by Golan A. et al in his research Congenital Anomalies of the Mullerian System. ${ }^{[7]}$

\section{Conclusion}

This study was done to help clinicians to better understand the reproductive, gestational and neonatal outcome in the case of uterus didelphys. Uterus didelphys is the rarest form of Mullerian duct defects and if it wasn't for the surgeon's inquisitive personality, we would have a common case of bicornate uterus instead of the rare defect of uterus didelphys.

\section{References}

[1].Three-dimensional ultrasound in the diagnosis of mullerian duct anomalies and concordance with magnetic resonance imaging. Bermejo C, Martinez Ten P, Cantarero R, Diaz D, Perez Pedregosa J, Barron E, Labrador E, Ruiz Lopez L.

[2].http://emedicine.medscape.com/article/273534-overview\#a2 (incidence and prevalence).

- Clinical implications of uterine malformations and hysteroscopic treatment results. Grimbizis GF, Camus M, Tarlatzis BC, Bontis J, Devroey P.

- Incidence of mullerian defects in fertile and infertile women. Acien P.

- Mullerian duct anomalies diagnosed by saline contrast sonohysterography: prevalence in a general population. Dreisler E, Stampe Sorenson S.

- Reproductive outcomes in women with uterine anomalies. J Women's Health (Larchmt). Lin PC.

[3].Uterine anomalies. How common are they, and what is their distribution among subtypes? Nahum GG.

[4].The obstetric consequences of uterovaginal anomalies. Rock JA, Sclaff WD.

[5].Endometroid adenocarcinoma arising in uteri with incomplete fusion of mullerian ducts. Reports of three cases. Kondi- Pafiti A, Spanidou- Carvouni H, Dimopoulou C, Kontogianni CI.

[6].Diagnosis of uterine anomalies: relative accuracy of MR imaging, endovaginal sonography and hysterosalpingography. Pellerito JS, McCarthy SM, Doyle MB, Glickman MG, DeCherney AH:1992.

[7].Congenital anomalies of the mullerian system. Golan A, Langer R, Bukovsky I, Caspi E. 